\title{
Norois
}

Environnement, aménagement, société

245 | 2017

Adapter les territoires aux changements climatiques : transition urbanistique et aménagement de l'espace

\section{La transition énergétique : des modèles diversifiés et contrastés à l'échelle régionale}

Le cas de l'Alsace dans la région du Rhin-Supérieur

Energy transition: diversified and contrasting models on a regional scale. The case of Alsace in the Upper Rhine region

\section{Guillaume Christen}

\section{(2) OpenEdition}

12 Journals

Édition électronique

URL : http://journals.openedition.org/norois/6231

DOI : $10.4000 /$ norois.6231

ISBN : 78-2-7535-7465-6

ISSN : $1760-8546$

\section{Éditeur}

Presses universitaires de Rennes

Édition imprimée

Date de publication : 31 décembre 2017

Pagination : 63-74

ISBN : 978-2-7535-7456-4

ISSN : 0029-182X

\section{Référence électronique}

Guillaume Christen, « La transition énergétique : des modèles diversifiés et contrastés à l'échelle régionale », Norois [En ligne], 245 | 2017, mis en ligne le 31 décembre 2019, consulté le 11 janvier 2021. URL : http://journals.openedition.org/norois/6231 ; DOI : https://doi.org/10.4000/norois.6231 
pour chaque région de réaliser un Schéma régional du climat, de l'air et de l'énergie (SRCAE) ${ }^{1}$.

Bien que les énergies renouvelables (l'éolien, le solaire thermique et photovoltaïque, la biomasse ou encore la géothermie) soient porteuses d'une possible reconfiguration des systèmes énergétiques centralisés (Rumpala, 2013), le regard des sciences sociales souligne les contours protéiformes de la transition énergétique. Ses orientations font donc l'objet d'une pluralité de traductions, voire apparaissent controversées, et témoignent d'une politisation de l'enjeu. L'approche sociologique examine les innovations écologiques, mais également les institutions et les acteurs qui soutiennent la transition. En effet, les acteurs collectifs sont porteurs de schémas d'interprétation (Lascoumes et Le Galès, 2005) : ils introduisent des conceptions de la transition énergétique qui définissent différemment les relations entre écologie et économie. Il s'agit à la fois de spécifier les réseaux d'acteurs qui composent les filières, et de qualifier les représentations que ceux-ci ont de la transition. Partagent-ils les mêmes grilles de lecture? Dans quelle mesure viennent-ils questionner la représentation d'une ressource inépuisable et, plus largement, amorcer - ou pas - un paradigme énergétique renouvelé? Notre propos s'appuiera sur une enquête de terrain réalisée à l'échelle de l'ancienne région Alsace dans le cadre d'un programme de recherche européen "Interreg Plan énergies renouvelables » (2012-2015). Le cas de ce territoire peut s'avérer fort exemplaire pour la problématique qui nous concerne, à commencer par la place du Rhin. En effet, la production d'hydroélectricité sur le Rhin représente actuellement $12 \%$ de la production française d'énergie renouvelable, avec une puissance de 1200 MW. Par ailleurs, on peut également évoquer le cas de Fessenheim² ${ }^{2}$ la première centrale nucléaire française qui a été construite sur le territoire national. Toujours en activité en 2017, la centrale fournit 2,1\% de la production d'électricité toutes sources confondues en France en 2010, et $88 \%$ de la consommation alsacienne en 2012 .

1. Loi n 2010-788 du 12 juillet 2010 portant engagement national pour l'environnement.

2. Situés au sud-est de Colmar et au sud-ouest de Fribourg-en-Brisgau, deux réacteurs d'une puissance de 900 MW chacun ont été édifiés entre 1971 et 1978. La construction de la centrale s'est appuyée sur la participation financière de trois entreprises du secteur de l'énergie : EDF $(67,5 \%)$, EnBW $(17,5 \%)$ et un consortium suisse (NOK, EOS et BKW) $(15 \%)$. Ces entreprises obtiennent un prélèvement de l'électricité proportionnel à leur participation financière.
La programmation de son possible arrêt soulève l'enjeu du développement d'énergies alternatives. Nous avons conduit des entretiens semi-directifs auprès des acteurs de la scène énergétique locale (plus d'une trentaine), tant au niveau des opérateurs privés (fournisseurs d'énergie, entreprises d'installation de panneaux solaires) que des associations et initiatives citoyennes. Plus précisément, notre propos s'est focalisé sur un projet de parc éolien dans la commune de $\mathrm{S}^{3}$, dont la particularité tient au financement participatif (de type actionnariat populaire) de deux éoliennes sur les huit construites. Nous avons mené sur place une enquête à partir d'entretiens semi-directifs ${ }^{4}$ auprès des porteurs de projet, qui ont participé à l'élaboration de l'actionnariat, en particulier l'adjoint chargé des questions environnementales et du dossier éolien à S., ainsi que le maire de la commune concernée. Au-delà, s'agissant d'approcher l'ensemble des acteurs impliqués au sein des réseaux associatifs d'énergie coopérative, nous avons rencontré des responsables de cinq collectifs, locaux et nationaux, investis dans le développement du même projet.

La première partie revient sur la dimension sociale d'un modèle énergétique. En effet, celui-ci inclut à la fois des acteurs, des machines, des techniques, qui donnent forme au collectif sociotechnique (Rumpala, 2013). En somme, un système énergétique repose sur des infrastructures et des réseaux de production et de distribution des flux d'énergies. Or, ces réseaux ne sont pas désincarnés, car les techniques et les installations mises en œuvre sont organisées par des collectifs d'acteurs qui gèrent les flux de ressources (Rumpala, 2013). Ainsi, le développement des énergies renouvelables ne signifie pas seulement le remplacement de sources d'énergie par d'autres, mais transforme l'ensemble du réseau qui les exploite et les diffuse (Évrard, 2014). Dès lors, la transition vers d'autres sources d'énergie ne va pas de soi et implique une dynamique sociale, qui interroge le rapport à la nature, à la technique et

3. La commune de S. est un village de moyenne montagne dans le département du Bas-Rhin, qui compte 847 habitants en 2011. Par rapport au contexte régional, elle se caractérise par des inégalités accrues : le taux de chômage des 15-64 ans est de 17,9\%, contre $10 \%$ pour la région Alsace, en 2009. En termes de niveau de revenu, on constate que le revenu net moyen déclaré par foyer fiscal est de $17292 €$, contre $26691 €$ pour l'ensemble de la région Alsace.

4. Nous avons bénéficié, pour une partie de ce travail, de l'appui d'Amandine Léonate, assistante-ingénieure contractuelle à l'UMR SAGE (Léonate, 2013). 
au politique (Raineau, 2011). À l'échelle régionale, nous reviendrons sur l'importance des décisions énergétiques passées, dont le poids conditionne les possibilités de changement, révélant les effets d'une « dépendance » (Pierson, 2000) autour du nucléaire civil. Quant à la deuxième section, elle revient sur les différentes lectures développées à la fois en termes d'« économie verte » (Hubert et Mormont, 2008), et de systèmes coopératifs ou citoyens. Nous verrons que la transformation se joue dans une tension entre rupture et continuité, répondant à différentes acceptions de la transition.

\section{UN POTENTIEL DE RECONFIGURATION LIMITÉ PAR DES CHOIX ANTÉRIEURS}

Bien que le passage d'un système d'énergies fossiles vers des ressources renouvelables soit porteur d'une possible reconfiguration des modèles (Rumpala, 2013), les choix passés conditionnent les orientations données à la "transition énergétique » (Theys, 2014). En effet, il n’y a pas là un cheminement unique et stable: il dépend des réseaux d'acteurs qui portent et diffusent les énergies renouvelables, faisant de la transition énergétique une «question plurielle et hétérogène » (Rutherford, 2013), conflictuelle en somme.

\section{L'inertie d'un système énergétique antérieur}

Les choix énergétiques ne relèvent pas exclusivement de dimensions techniques, mais l'exploitation de telle ou telle source d'énergie et sa distribution renvoient à des choix politiques et de société (Rumpala, 2013). Au demeurant, un système énergétique repose sur des collectifs sociotechniques, c'est-à-dire des spécialistes s'appuyant sur des savoirs et des référentiels techniques. Le système énergétique français, qui s'est bâti autour du nucléaire civil, renvoie à une production essentiellement centralisée, organisée en filières (Debourdeau, 2011), selon des circuits longs, encadrés en amont et en aval par des réseaux experts (Évrard, 2014). En effet, le secteur du nucléaire civil représente un domaine professionnel qui repose sur une légitimation de savoirs techniques comme vecteurs de confiance et de progrès. Il s'est constitué autour d'un collectif sociotechnique composé de trois principaux acteurs : la Direction générale de l'éner- gie et des matières premières (DGEMP), Electricité de France (EDF) et le Commissariat à l'énergie atomique (CEA), qui forment un espace social relativement clos, au sein duquel s'échangent, par le biais de relations fréquentes, l'expertise et l'influence sur les décisions en matière de politique électrique d'origine nucléaire (Évrard, 2014). À compter de 1983, ce collectif s'élargit avec la création de la Société des participations du CEA, dénommée CEA-Industrie. En septembre 2001, CEA-Industrie fusionne avec deux autres entités, la COGEMA ${ }^{5}$ et Framatome ${ }^{6}$, sous le nom d'Areva $^{7}$, afin de fonder un groupe intervenant sur l'ensemble de la chaîne nucléaire, de l'extraction du combustible à la construction des centrales et au traitement des déchets. Au-delà de la chaîne d'exploitation, les systèmes énergétiques influencent les sociétés vers des modèles particuliers. Alain Gras (2007) qualifie de «thermo-industrielles » les sociétés fondées sur un modèle d'énergies fossiles. De son point de vue, les sociétés industrielles sont conçues à partir du même procédé du feu, qui renvoie à l'énergie tirée et transformée à partir de la combustion des ressources fossiles : pétrole, gaz naturel, charbon, uranium, etc. De la sorte, un schéma énergétique reposant exclusivement sur l'énergie fossile a modelé le rapport que les acteurs entretiennent aux ressources. Dès lors, une énergie produite à partir de sources fossiles ou renouvelables ne renvoie pas aux mêmes imaginaires ni aux mêmes modèles de société (Raineau, 2011). Ces deux modes de production contribuent, au contraire, à des rapports différents au monde et à la nature. L'indépendance acquise à l'aide des énergies fossiles a rendu possible le fait de se soustraire aux aléas naturels et a conduit à une programmation continue de la distribution d'énergie (Gras, 2003) : la production d'énergie était désormais contrôlée et adaptée aux besoins de consommation, de façon à satisfaire la demande (Coutard et Rutherford, 2009).

\footnotetext{
5. La Compagnie générale des matières atomiques (COGEMA) est spécialisée dans le recyclage des combustibles usés et le démantèlement des installations nucléaires.

6. La société Franco-américaine de constructions atomiques (Framatome) a été fondée en 1958 par les entreprises Schneider, Merlin Gerin et Westinghouse, et poursuit ses activités à partir de 1999 dans le cadre d'Areva Nuclear Power.

7. La principale activité d'Areva est l'exploitation de l'énergie nucléaire, à partir d'une organisation en cinq pôles : le groupe mine (extraction), le groupe amont (enrichissement de l'uranium et conversion en combustible), le groupe réacteurs et services (conception et fabrication des réacteurs), le groupe dédié au développement du réacteur EPR, et le groupe aval (recyclage et traitement des combustibles).
} 
L'image d'un métabolisme linéaire a été souvent évoquée (Coutard, 2001, 2010) pour figurer un tel modèle interconnecté qui planifie d'une manière centralisée les objectifs à atteindre. Il fonctionne selon un flux continu d'approvisionnement de matières, de production d'énergie et d'élimination des déchets. À l'opposé, un réseau décentralisé fonctionne selon un métabolisme circulaire et court (tableau 1).

Le développement d'un réseau technique est également susceptible de générer une "idéologie du réseau »(Coutard, 2001). La croyance en ce mode de régulation légitime l'idée selon laquelle les solutions aux problèmes sont à rechercher dans les caractéristiques du réseau lui-même, à savoir plus de centralisation, d'extension et de technique. L'intégration des énergies renouvelables dans le modèle centralisé en tant qu'énergies de substitution s'analyse comme une réponse du réseau centralisé face à la crise écologique, en confirmant un cadre qui reste inchangé. Dans cette perspective, l'entrée des énergies alternatives est-elle susceptible de conduire à une redistribution du schéma existant et implique-t-elle des possibilités de changement social? Dès lors, le véritable enjeu se situe autour du degré de rupture des innovations énergétiques avec un modèle centralisé.

\section{La transition énergétique : continuité ou rupture avec le modèle existant?}

Nous avançons l'hypothèse que l'introduction et le développement de technologies alternatives (éoliennes, énergie solaire...) ouvrent des possibilités

\begin{tabular}{|c|c|}
\hline $\begin{array}{c}\text { Métabolisme linéaire ou } \\
\text { réseau centralisé }\end{array}$ & $\begin{array}{c}\text { Métabolisme circulaire ou } \\
\text { réseau décentralisé }\end{array}$ \\
\hline $\begin{array}{c}\text { Production - approvision- } \\
\text { nement - élimination des } \\
\text { déchets }\end{array}$ & Réutilisation - recyclage \\
\hline Cycle long & Cycle court \\
\hline Modèle de flux continu & Logique de stock \\
\hline $\begin{array}{c}\text { Logique d'offre - production } \\
\text { - satisfaction de la demande }\end{array}$ & Modèle écologique \\
\hline $\begin{array}{c}\text { Modèle technico-écono- } \\
\text { mique }\end{array}$ & \\
\hline
\end{tabular}

Tableau 1 : Caractéristiques d'un système énergétique à partir d'un métabolisme linéaire vs circulaire (Coutard, 2001).

Characteristics of a energy systems based on a linear metabolism vs a circular metabolism nouvelles, susceptibles d'infléchir, en partie du moins, les modèles en place. En effet, le potentiel de changement des énergies renouvelables n'est pas porté par la technique seule. Au contraire, les pistes de changement trouvent leur origine dans les orientations que les acteurs donnent aux technologies alternatives (Akrich, 1993), c'est-à-dire qu'elles résident essentiellement dans la manière dont les acteurs pilotent les technologies nouvelles et la signification sociale qu'ils leur accordent. En ce sens, la notion de «potentialité technique » présentée par James J. Gibson (1979) permet de penser ensemble la technique et le social, en désignant à la fois ce qu'une innovation technique offre comme prise sur les écosystèmes et les formes d'appropriation élaborées par les acteurs. C'est dire que les sources d'énergies renouvelables, ne portent pas intrinsèquement un potentiel de changement, celui-ci dépend surtout des orientations choisies par les acteurs. Frank Adler et Ulrich Schachtschneider (2010), qui proposent une typologie des solutions au réchauffement climatique, constatent que s'il existe aujourd'hui un relatif consensus sur la réalité de la crise écologique, ses causes et a fortiori les réponses, sont quant à elles controversées. Dans cette perspective, Karl Werner-Brand (2014) a classé les solutions possibles selon deux idéaux-types susceptibles d'apporter un éclairage intéressant quant aux orientations que la transition énergétique est susceptible de prendre :

- La transformation fondamentale du système actuel (modification du système de valeurs);

- La modernisation dans le cadre du système actuel (accent mis sur les innovations techniques);

Dans la première orientation, la crise écologique est la conséquence directe de notre modèle socioéconomique et culturel, et, pour répondre à la crise, il faut le modifier radicalement. La réponse doit être organisée selon un principe de solidarité et d'égalité, et cherche à développer les démarches citoyennes et collectives. La forme organisationnelle et le rapport aux ressources diffèrent en mettant en avant un principe d'éco-centrisme qui réévalue les cycles naturels ainsi que le rôle et la fonction auxiliaire des objets de la nature. À l'inverse, le second modèle, basé sur l'économie de marché, est dit « technocentré ». Le développement durable constitue une nouvelle «niche " économique, où les questions environnementales sont intégrées et régulées par le marché. Dans cette configuration, l'innovation 
technique devient le moteur d'un développement « durable », dès lors qu'elle permet de déployer des modes de production moins énergivores, et définit une économie bas carbone. La notion de modernisation écologique, développée par Joseph Huber (1982), décrit comment les risques environnementaux et les solutions pour y répondre sont définis autour de registres d'action délimités, suivant l'hypothèse selon laquelle les sociétés industrielles ont intégré l'environnement dans une dynamique de conciliation entre le progrès technique et le marché économique. À ce titre, la modernisation écologique marque, selon Edwin Zaccai (2010), l'expression de la poursuite de la modernité. Suivant cette perspective, les réponses au réchauffement climatique résident dans le développement de technologies performantes, dans le but de réduire la consommation d'énergie. Ceci marque l'importance de l'«éco-innovation » (Coulbaut-Lazzarini et Némoz, 2013) : il s'agit de générer des opportunités de profits à partir d'innovations techniques visant à atténuer les dégradations des ressources par la création d'une « économie verte ». Intégrée au projet de modernisation écologique, la transition énergétique s'inscrit dans une transformation continue et organisée et ne renverse pas le modèle établi : elles substituent aux énergies fossiles des sources renouvelables, sans toucher au cadre existant (Rudolf, 2013).

La transition énergétique se décline via différents processus qui ne sont pas univoques et demeurent instables (Raineau, 2011) : il existe «des » transitions énergétiques. Les sources d'énergies renouvelables se caractérisent par leur «flexibilité » (Zélem, 2012), et sont susceptibles de s'adapter et de s'intégrer différemment selon les modes de régulation. À partir du territoire du Rhin Supérieur et plus particulièrement du cas alsacien, nous proposons d'observer la façon dont les acteurs (opérateurs industriels, initiatives citoyennes) mettent en place des projets différenciés, voire controversés de déploiement des énergies renouvelables.

\section{Des transitions énergétiques}

Une même source d'énergie peut être porteuse de transitions énergétiques différentes, socialement et territorialement, en fonction des collectifs d'acteurs qui la portent. Autrement dit, les énergies renouvelables peuvent se développer soit comme éner- gie de substitution dans un système centralisé, soit sous des formes coopératives territorialisées, où se constituent des modes collectifs de gestion décentralisés. Mais la description de ces modes de régulation nécessite de revenir plus en amont sur des choix historiques qui conditionnent le déploiement des énergies renouvelables à l'échelle territoriale.

\section{LA TRANSITION ÉNERGÉTIQUE À L'ÉCHELLE RÉGIONALE}

En Alsace, les enjeux énergétiques retraduisent à la fois les objectifs européens de la transition (les « $3 \times 20^{8}$ », à atteindre d'ici 2020, et le «facteur $4^{9}$ », d'ici 2050) ainsi que les effets du cadre national façonné autour du nucléaire civil. La loi Grenelle II fait obligation pour chaque région de réaliser un Schéma régional du climat, de l'air et de l'énergie (SRCAE) ${ }^{10}$. Pour l'Alsace, il s'agit d'atteindre, par des sources alternatives, $26 \%$ de la consommation totale d'énergie d'ici 2020. La figure 1 traduit les répercussions des choix historiques tournés vers l'hydraulique et le nucléaire civil en Alsace : ce dernier, incarné dans la centrale de Fessenheim, correspond à 72,2\% de l'énergie primaire produite en 2010 , et les aménagements hydrauliques sur le Rhin 16,1\%.

Le cas alsacien ne fait pas exception par rapport à la situation nationale, où l'énergie provient essentiellement du nucléaire civil, à hauteur de $93 \%$ de l'énergie primaire non renouvelable produite. De même, les objectifs du SRCAE et le potentiel mobilisable illustrent un déséquilibre entre secteurs énergétiques : en 2010, $90 \%$ des énergies renouvelables produites sont issues de l'hydroélectricité $(69,5 \%)$ et de la biomasse-bois (22\%) (figure 2). L'énergie solaire reste marginale, tout comme l'éolien : le solaire photovoltaïque et le solaire thermique ne pèsent ensemble que $4 \mathrm{Ktep}^{11}$ en 2009 , soit $0,4 \%{ }^{12}$

8. La règle des « $3 \times 20$ » demande d'atteindre d'ici à 2020 une réduction de $20 \%$ des émissions de gaz à effet de serre, une augmentation de $20 \%$ de la part des énergies renouvelables (hydroélectricité, solaire, éolien, biomasse ou géothermie) dans la consommation énergétique totale de l'Union européenne.

9. Le facteur « 4 » évoque la division par quatre d'ici à 2050 des émissions de gaz à effet de serre, sur la base des chiffres de 1990

10. Loi $\mathrm{n}^{\circ} 2010-788$ du 12 juillet 2010 portant engagement national pour l'environnement.

11. KTep : kilo tonne équivalent pétrole est une unité de mesure utilisée pour exprimer la valeur de production énergétique de chaque matière.

12. Source : rapports Énergivie-info/CREA (Conférence régionale de l'énergie et de l'atmosphère en Alsace) 


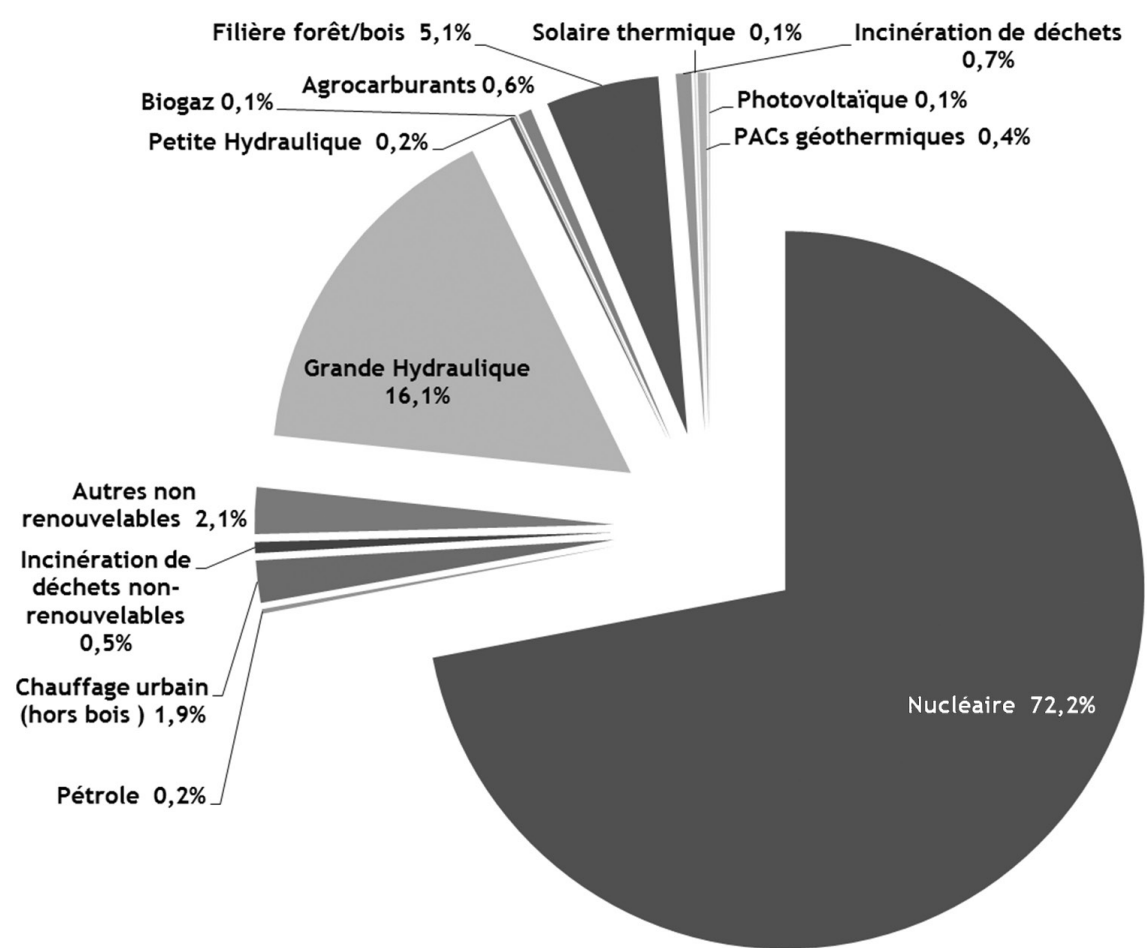

sources: CREA. Alsace
Figure 1 : Répartition de toutes les énergies dans la production d'énergie primaire en Alsace en 2010

Distribution of all types of energy in primary energy production in Alsace in 2010

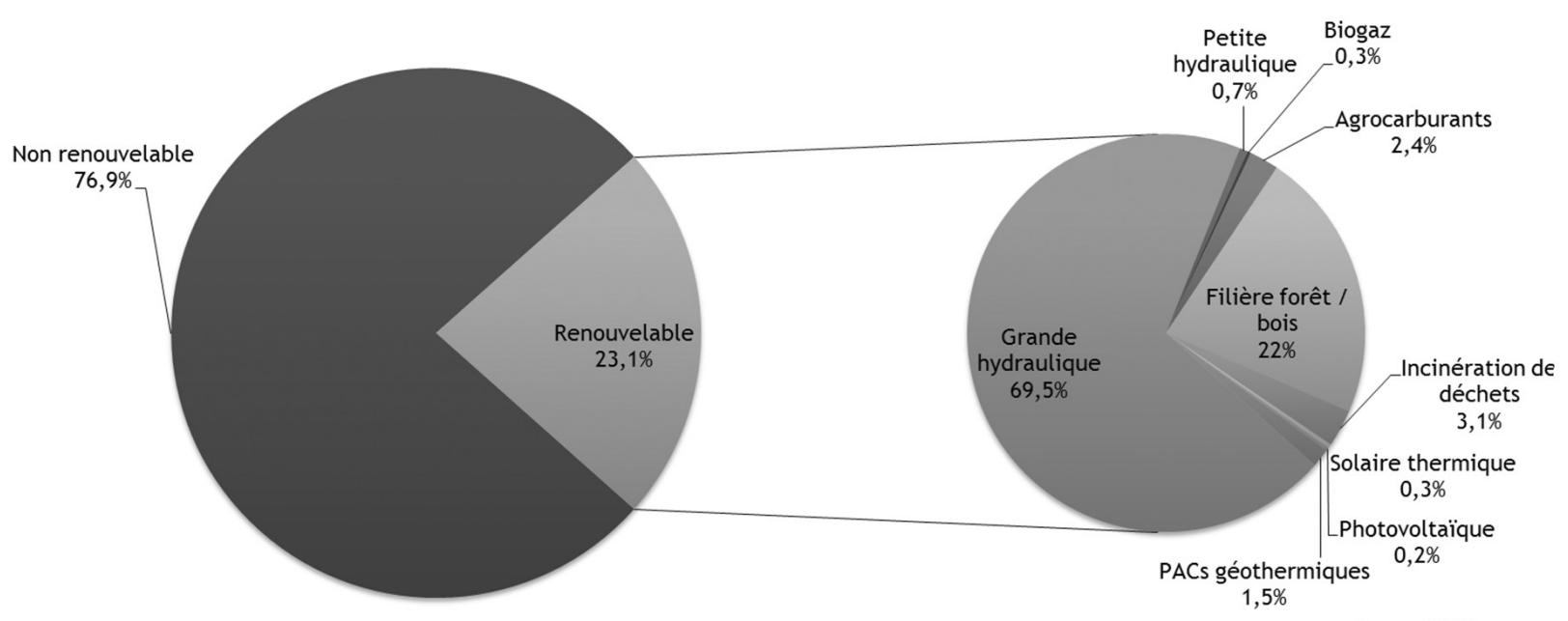

Source : CREA

Figure 2 : Répartition des énergies renouvelables dans la production d'énergie primaire en Alsace en 2010 Distribution of renewable energy sources in primary energy production in Alsace in 2010

du total des ENR, quant à l'énergie éolienne en 2009 sa production est inexistante (tableau 2).

Les filières bois et hydraulique apparaissent désormais saturées, conduisant les pouvoirs publics à promouvoir à présent une diversification du mix énergétique. En effet, la mise en œuvre du Grenelle de l'environnement a induit un fort développement du bois-énergie en Alsace. Le « discours d'Urmatt », prononcé le 19 mai 2009 par le président Nicolas Sarkozy dans cette commune du Bas-Rhin ${ }^{13}$, fait de la filière bois un pivot de la transition énergétique, devant supporter à elle seule plus du tiers de l'augmentation de la part d'énergies renouvelables dans la consommation énergétique française d'ici 2020, soit

13. Discours disponible en ligne : http://agriculture.gouv.fr/IMG/pdf/discours-filiere-bois_19mai09_cle864281.pdf (consulté le 16 février 2015). 


\begin{tabular}{|c|c|c|}
\hline $\begin{array}{c}\text { Filière de } \\
\text { production }\end{array}$ & $\begin{array}{c}\text { Production } \\
2009 \text { (ktep) }\end{array}$ & $\begin{array}{c}\text { Potentiel de } \\
\text { production } \\
2020 \text { (ktep) }\end{array}$ \\
\hline Hydroélectricité & 650 & 660 \\
\hline Biomasse-bois & 214 & 266 \\
\hline Biomasse déchets & 32 & 50 \\
\hline Biomasse agricole & 0 & 5 \\
\hline Agrocarburants & 23 & 30 \\
\hline Géothermie profonde & 0 & 20 \\
\hline Géothermie de surface & 12 & 26 \\
\hline Solaire thermique & 3 & 24 \\
\hline Solaire photovoltaïque & 1 & 28 \\
\hline Biogaz & 3 & 12 \\
\hline Éolien & 0 & 20 \\
\hline Total & 938 & 1141 \\
\hline $\begin{array}{c}\text { Production d'énergies renou- } \\
\text { velables sur la consommation } \\
\text { finale }\end{array}$ & $17,4 \%$ & $26,5 \%$ \\
\hline
\end{tabular}

Tableau 2 : Production d'énergies renouvelables en Alsace en ktep/ an en 2009 et potentiel de production en 2020 (source : rapports Énergivie-info/CREA)

Renewable energy production in Alsace in ktoelyear in 2009 and production potential in 2020

21 millions de $\mathrm{m}^{3}$ de plus de production annuelle de bois. Outre les mesures destinées aux particuliers, d'autres outils ont favorisé l'expansion d'une filière industrielle autour du bois-énergie, à commencer par la simplification des procédures d'appel d'offres de la Commission de régulation de l'énergie (CRE) pour les unités industrielles de production d'électricité à partir de biomasse. Les pouvoirs publics appuient ainsi la filière biomasse-bois, déjà fortement sollicitée, en développant des réseaux de chaleur à taille industrielle. L'hydraulique affiche elle aussi des possibilités de croissance restreintes, dans la mesure où elle représente déjà $69,5 \%$ de la production d'électricité primaire en 2010. Dix centrales hydrauliques, construites entre 1932 et 1970, fonctionnent sur le Rhin et sont principalement exploitées par EDF, ou sous la forme d'un partenariat entre EDF et l'électricien allemand EnBW (pour Gambsheim). Or, dans un souci de mix énergétique, les objectifs à 2050 prévoient une nette diminution de la part de l'hydroélectricité et un équilibre de la biomasse, au profit du solaire thermique et photovoltaïque (10\% de la production primaire des énergies renouvelables), ainsi que de l'éolien (4\%).

Le SRCAE tente de mobiliser des énergies renouvelables encore peu exploitées. C'est le cas de la géothermie, de la biomasse ou encore de l'éolien, ainsi que du solaire thermique et du solaire photovoltaïque - secteurs qu'il est prévu de soutenir pour parvenir à $30 \mathrm{ktep}$ de production d'ici 2020 (tableau 2). On le saisit, les disparités entre secteurs d'énergie renouvelable dessinent des déséquilibres qui traduisent l'historicité des modèles et des politiques énergétiques (Évrard, 2014). Les sources renouvelables dont l'exploitation a été développée, sont celles qui s'intègrent au sein des circuits existants, c'est-à-dire des réseaux longs et centralisés. C'est particulièrement le cas pour l'hydroélectricité (l'énergie produite est réinjectée dans le circuit existant) ainsi que les réseaux de chaleur à destination des habitats collectifs. Dans ce cas, le réseau géré par un opérateur est converti en renouvelables (vers la biomasse) et la chaleur produite revendue au bailleur social. Dans ce type de configuration, les énergies renouvelables sont intégrées aux réseaux centralisés existants, où l'on attend d'elles les mêmes logiques et fonctions que les sources d'énergies fossiles.

\section{Des transitions énergétiques ou vers une hybridation des modèles?}

À partir d'enquêtes de terrain auprès des acteurs clés territoriaux à l'échelle du Rhin Supérieur, nous pouvons définir une typologie des modes de régulation et des orientations données à la transition énergétique. En effet, la transition énergétique s'opère concrètement en France à partir de deux modes d'introduction opposés (Christen et al., 2013) : une régulation par le marché, largement dominante, et une «alternative » portée par des initiatives qui se présentent comme citoyennes. Elles renvoient à des intégrations différenciées des technologies énergétiques, qui traduisent la tension entre deux modèles « soft » et « hard path » (Évrard, 2014) : le second correspond à une régulation centralisée-industrielle et le premier représente des systèmes décentralisés à l'initiative d'un secteur associatif développant une régulation citoyenne de l'énergie.

\section{Une régulation par le marché}

La diffusion des énergies renouvelables dans la société peut s'effectuer principalement par le marché, afin de développer un secteur industriel 
(Debourdeau, 2011). Elle s'appuie essentiellement sur le déploiement d'innovations énergétiques que nous pouvons qualifier d' « éco-innovation » (Coulbaut-Lazarinni et Némoz, 2013). Ces innovations cherchent à générer des opportunités de profits via la création d'une "économie verte " (Scarwell, 2012). Ce sont de véritables «packs » (Debourdeau, 2011) que les opérateurs industriels proposent aux particuliers, aux collectivités ou encore aux bailleurs sociaux. Ceux-ci comprennent les études préalables, l'installation du système, la maintenance, le rachat ou la vente de l'énergie produite à l'image des réseaux de chaleur à destination des habitats collectifs. À titre d'exemple, le réseau de chaleur du quartier de la «Cité de l'Ill » (au nord de l'Eurométropole de Strasbourg) a été traditionnellement délégué à des opérateurs du secteur des énergies : Shell, Dalkia, et désormais Idex depuis 2000. Ce même prestataire a été retenu pour l'exploitation de la centrale à cogénération dans le cadre de la conversion de la chaudière. Les prestations de l'entreprise englobent : l'approvisionnement, la production et la distribution de la chaleur ainsi que l'entretien du réseau. Dans cette délégation de service, l'opérateur revend au bailleur la chaleur produite suivant un contrat qui fixe un prix au kWh.

Si l'on considère l'échelle régionale, on remarque que la mise en œuvre de la transition énergétique repose principalement sur des opérateurs historiques (Électricité de Strasbourg, Dalkia, etc.) qui bénéficient de la gestion d'un réseau existant (chaleur, électricité). Dans le cas de l'Eurométrople de Strasbourg, la production d'électricité « verte » est essentiellement captée par un opérateur local, en l'occurrence Électricité de Strasbourg. Quant au réseau de chaleur urbain, on peut citer la société Dalkia qui pilote les quartiers de Hautepierre et de l'Esplanade. Le positionnement d'opérateurs industriels a été soutenu par les appels d'offres initiés par la Commission de régulation de l'énergie (CRE) à l'échelle nationale. L'appel lancé en 2009 a porté sur une puissance de 250 MWc à partir de biomassebois. Deux projets ont été retenus en Alsace, pour une puissance de $14 \mathrm{MWc}$ : une centrale à cogénération portée par Dalkia ${ }^{14}$, et une seconde par la scierie SIAT-Braun à Urmatt (Bas-Rhin) pour une puissance de $4 \mathrm{MWc}$. Les énergies "vertes» se

14. Dalkia est une filiale de Véolia Environnement et d'EDF. Le projet vise à alimenter un réseau de chaleur du quartier de l'Esplanade à Strasbourg. substituent ici aux énergies fossiles dans le réseau sociotechnique en place. Cette captation de la transition énergétique par la « cité marchande » (au sens de Lafaye et Thévenot, 1993) incarne le projet d'écologie industrielle. Dans ce type de configuration, on observe l'émergence d'une « technologie verte » (Hubert et Mormont, 2008), c'est-à-dire un ensemble d'innovations dont les buts sont d'atténuer ou de réparer les dégradations des ressources naturelles. Ces techniques « vertes » prolongent une régulation centralisée autour d'acteurs économiques dominants et illustrent un "impensé impensable » (Évrard, 2014) : le développement des énergies renouvelables est immédiatement capté et conforme à l'organisation du modèle centralisé, grâce aux ressources dont dispose le secteur industriel. On peut dresser le constat selon lequel les énergies renouvelables sont largement absorbées par le secteur industriel (Évrard, 2014).

\section{Le développement des coopératives citoyennes}

En Allemagne, dès la fin de la décennie 1980, les énergies renouvelables se diffusent de manière innovante à partir d'initiatives citoyennes, telles des coopératives énergétiques. Leur développement s'est accéléré, de sorte que l'on compte en 2012 plus de 750 installations gérées en coopérative (Poize et Rüdinger, 2014), tels des toits solaires ou des éoliennes citoyennes.

En Alsace, différents projets participatifs ont émergé pour développer des petites unités de production d'électivité « verte » à partir de solaire photovoltaïque ou de microcentrales hydroélectriques. Le toit photovoltaïque et collectif est l'outil privilégié, à l'image de l'association Énergies citoyennes d'Alsace (ECA) fondée en 2008 près de Thann pour l'installation de $200 \mathrm{~m}^{2}$ de panneaux avec une puissance de 27,91 kWc. Les membres fondateurs d'ECA sont issus d'une organisation environnementale militante locale, "Thur écologie transport », qui a organisé en juillet 2007 un déplacement dans la commune de Freiamt près de Freiburg, en Allemagne, où la production d'énergie est à $75 \%$ décentralisée; cette visite a été le déclencheur du projet.

Une difficulté a été l'impossibilité de bénéficier d'un toit public : "On voulait faire une installation sur un toit public mais personne ne nous a soutenus. On avait une possibilité sur la vallée dans un musée 
textile à Wesserling, mais on s'est rendu compte que l'emplacement est trop encaissé, il y a un château d'eau qui fait de l'ombre, différentes contraintes faisaient que ce n'était pas favorable " (entretiens, président d'ECA à Leimbach, décembre 2012 et janvier 2013). Le collectif a alors retenu un toit privé, qu'il loue auprès d'un particulier dans le village d'Aspach-le-Bas. Le financement du toit solaire a été rendu possible par l'engagement des 42 associés (soit 30000 euros), qui ont chacun investi la même somme, hormis le propriétaire, solidaire à hauteur de $33 \%$. Le montage financier a supposé la création d'une nouvelle structure associative : Soleil citoyen d'Aspach-le-Bas. Il repose sur un emprunt de 85000 euros et une subvention de 20000 euros de la Région Alsace. Quant aux dividendes versés, il a été décidé de les fixer sur le taux de rémunération du livret A. Un membre de l'association y insiste, les motivations ne relèvent pas du profit économique, mais d'un engagement militant : "On a acté dans une Assemblée générale que les dividendes ne seraient pas supérieurs au livret A. On ne vient pas chez nous pour faire du $8 \%$ de rentabilité, on le fait pour développer des énergies renouvelables! (entretien, membre de l'association Soleil citoyen d'Aspach-leBas, janvier 2013).

Le pilotage de ce type de projet requiert des compétences techniques et professionnelles spécialisées. Pour y faire face, les collectifs sont organisés en mode fédératif, facilitant le transfert et le partage de connaissances. Chaque structure locale bénéfice de compétences en réseau, c'est-à-dire des formes de savoirs et d'expériences pratiques acquises et entretenues par les uns et les autres. On a pu observer un réseau associatif qui bénéficie d'une expertise collective en la matière. Les réseaux Ercisol ${ }^{15}$ et Alsace Énergie Partagée sont les relais régionaux d'Énergie Partagée ${ }^{16}$, association fédérative qui compte plus d'une cinquantaine de structures en France et dont l'objet est la promotion des énergies renouvelables et des modèles « citoyens » de la transition énergétique (éoliennes, centrales hydroélectriques...).

Témoin de cette organisation fédérative et en réseau, l'association Ercisol est sollicitée aussi bien par des particuliers que des collectivités pour

15. Énergies renouvelables citoyennes et solidaires, agréée Économie sociale et solidaire (ESS), qui a pour vocation la production de chaleur et d'électricité d'origine renouvelable.

16. [http://energie-partagee.org/] (consulté le 23 juin 2017). l'étude de projets (potentiel, faisabilité...), signe d'une reconnaissance désormais acquise en termes d'ingénierie, comme le précise le président d'énergie citoyenne d'Alsace : «L'association Énergie citoyenne d'Alsace est adhérente à Ercisol, on s'est mis avec eux car c'est une structure plus costaud, plus équipée pour faire des projets. On a des participants de tout l'Est. » L'association Ercisol, Énergies Renouvelables Citoyennes et Solidaires, créée en 2010, est une société par actions simplifiées qui encourage le mouvement des coopératives citoyennes de production d'énergies (électricité et chaleur) dont la source est d'origine renouvelable. Elle propose aux habitants de devenir actionnaires de projets locaux d'installation de microcentrales hydroélectriques, de centrales photovoltaïques (toits solaires) ou de parcs éoliens citoyens. L'association travaille sur différents projets et bénéficie d'expériences antérieures pour asseoir sa position, grâce aux liens tissés sur l'ensemble du territoire français et la gestion de plusieurs sites. À titre d'exemples, le collectif a piloté les projets suivants : une centrale photovoltaïque à Ebersheim (68), une centrale hydraulique à Nérac (47), et une centrale hydraulique à Moyenmoutier (88). La diversité des projets traduit l'importance de l'expertise collective qu'elle met au bénéfice des associations locales et fédérées.

\section{Vers une hybridation des modèles}

La technologie éolienne permet d'illustrer les relations possibles entre des logiques industrielles et des initiatives citoyennes, c'est-à-dire des possibilités contradictoires qui en font un « objet en transition » (Zélem, 2012). Dans le cas de S., nous abordons une forme hybridée de ces deux modèles, qui emprunte à la fois à la culture des coopératives énergétiques (autonomie, décentralisation de la production) et au cadre centralisé (alimentation et distribution de l'énergie dans le réseau global). Le site éolien de S. s'appuie sur un dispositif technicoéconomique introduit par un opérateur industriel et, adossé à celui-ci, deux éoliennes sur les dix construites, pour une puissance de 2 MW chacune, seront financées via une démarche coopérative d'actionnariat, pilotée par Énergie partagée ${ }^{17}$,

\footnotetext{
17. L'association Energie Partagée, crée en 2010, vise à essaimer les projets d'énergies renouvelables citoyens. Elle a également un rôle d'intervenant auprès des collectivités afin de leur présenter le modèle de projet citoyen
} 
une association spécialisée au niveau national. Une zone d'implantation de l'éolien terrestre (ZDET) a été déposée en 2008 concernant six communes des départements des Vosges et du Bas-Rhin. Deux modalités d'organisation se répondent ici. Suivant la première, la commune possédera $40 \%$ des parts, afin d'appuyer l'idée d'une territorialisation de la production d'énergie, qui, dans les faits, sera purement symbolique, la production étant réinjectée dans le réseau EDF. Mais les élus insèrent ainsi l'éolien dans une démarche territoriale, à travers un projet de développement local et touristique qui serait en même temps «durable »; ils transforment la technique éolienne pour lui donner une dimension collective, à la fois d'utilité locale et de bien commun environnemental (Christen, 2014). La deuxième médiation prend la forme d'un actionnariat populaire, défini par les porteurs du projet comme la possibilité donnée aux citoyens d'acquérir des actions des deux éoliennes à hauteur de $60 \%$. Là encore, la référence territoriale est ambiguë : si l'on affirme s'adresser aux habitants de S., en fait tout un chacun peut, moyennant l'aval de l'association gestionnaire, souscrire des parts via un site Internet, sans résider ni sur place ni en Alsace.

À l'image du dispositif qui est hybride, les acteurs qui le portent et l'organisent bénéficient également d'une double culture. Ainsi, d'un côté, l'appropriation citoyenne de la problématique énergétique est affirmée comme militante, au sens où les énergies renouvelables offriraient la possibilité d'une autonomie énergétique territorialisée face aux grands groupes industriels; et, de l'autre, on repère une rhétorique managériale, où le président d'Alsace Energie Partagée se présente comme un expertcommercial de cette même énergie « citoyenne »: «On est présent sur différents salons et forums, ou des conférences, on est déjà intervenu à la foire éco-bio de Colmar, on fait aussi des salons plus professionnels, comme le salon de l'habitat et de l'énergie à Blotzheim ou la Cité de l'habitat à Dornach où on a tenu des stands, mais là c'est plus ponctuel ${ }^{18}$. » L'acteur mobilise la figure composite du «techni-mili » (Nonjon,

et de les accompagner dans l'organisation pratique d'une coopérative. Le conseil d'administration est composé des associations régionales qui jouent un rôle de relais territorial. Parmi ces personnes morales on peut citer les associations « éoliennes en pays de Vilaine ", les « amis d'Enercoop », « Alsace énergie partagée », ou encore « Ercisol ».

18. Entretien réalisé avec le président de l'association Alsace énergie partagée, décembre 2012
2005), expert et militant, afin de « contourner et de se différencier de la posture froide et lointaine de l'expert technique » (Nonjon, 2005). De la sorte, la professionnalisation des associations d'énergie coopérative déplace les répertoires d'intervention de la militance vers la consultance, en termes d'ingénierie sociale, afin de piloter les projets d'actionnariat.

L'éolien citoyen illustre bien les possibilités contradictoires qu'offrent les technologies énergétiques. Un dispositif associatif et participatif, dont l'ambition est la promotion d'une appropriation citoyenne de l'énergie, cohabite avec un parc industriel dont la gestion est déléguée à un opérateur privé (Christen, 2014). Ce cas montre comment les configurations d'acteurs peuvent intégrer diversement les sources d'énergies renouvelables et donner des «styles techniques » (Akrich, 2006) différenciés à la transition énergétique. De même, la « recentralisation »des initiatives citoyennes autour d'acteurs industriels dominants traduit le caractère mouvant et instable des modèles énergétiques. Ceux-ci oscillent entre décentralisation et recentralisation, suscitant des formes transitoires et hybrides.

\section{Conclusion}

Au regard des orientations contradictoires, on remarque que la transition énergétique apparaît comme une "question plurielle et hétérogène " (Rutherford, 2013) où les technologies énergétiques se caractérisent par leur flexibilité (Akrich, 1999). En effet, les collectifs sociotechniques peuvent alors donner à ces technologies une orientation qui renforce la croissance verte (Brives et Mormont, 2008). Il est question de faciliter le développement d'innovations technologiques devant permettre d'atteindre des modes de vie moins énergivores, sans incidence sur l'économie. Dès lors, l'introduction des énergies renouvelables comme énergies de substitution témoigne de la capacité des opérateurs industriels à fournir des réponses face à la crise écologique, en confirmant un cadre qui reste inchangé.

On a observé en Alsace une prise de contrôle de ces enjeux par les acteurs spécialisés et un renforcement des opérateurs historiques de fourniture de services énergétiques. Parmi ces « alliances vertes » (Grolleau et al., 2008), en l'occurrence un arrangement entre économie de marché et enjeux climatiques, on peut citer la captation de la filière bois- 
énergie par les gestionnaires des réseaux de chaleur, dont la reconversion vers des sources renouvelables a principalement reposé sur les industriels et prestataires existants. Ces derniers maintiennent leur position dominante en intégrant le discours sur la durabilité et la demande environnementale en termes d'énergies renouvelables; ceci est corrélé à des politiques de transition énergétique qui se manifestent essentiellement «par l'offre plutôt que par la demande, politiquement incitée par des subventions, taxes ou autres mesures » (Wintz et Hajek, 2014). Une telle définition du processus transitionnel par l'évolution du «bouquet » énergétique élude la dimension, pourtant socialement importante, des habitudes de consommation et des modes de vie.

À l'opposé, le processus transitionnel peut favoriser une gestion collective par une réappropriation citoyenne de l'énergie comme un bien commun. En effet, certains collectifs tentent de penser et de pratiquer la transition énergétique en dehors d'une régulation par le marché, à l'exemple de coopératives ou d'associations nationales telles que Énergie partagée ou encore Alsace énergies partagées. Ces acteurs collectifs revendiquent une autonomie symbolique par rapport aux grandes compagnies d'énergie en favorisant de petites unités de production. Or, le couplage entre production et consommation locale de l'énergie apparaît marginal et contrarié. D'une part, les projets « citoyens », tels que la construction de toits solaires collectifs, ne seraient pas économiquement viables sans le tarif d'achat préférentiel mis en place par les pouvoirs publics. De l'autre, la production coopérative d'électricité "verte », justifiée par une volonté de sortir d'une régulation par le marché, reste dépendante des industriels lorsque l'énergie est réinjectée dans le réseau global.

Bien qu'affichant une ambition de rupture avec le modèle énergétique centralisé, dans les faits et à l'échelle des espaces concrets, le modèle coopératif repose également sur des compromis pratiques avec les logiques industrielles, dans la mesure où il est mis en œuvre selon les règles du jeu du système dominant (Alter, 1996). La faisabilité technique des projets « citoyens » étudiés tient à leur intégration partielle aux réseaux centralisés, et la garantie économique repose sur la revente de l'électricité à un opérateur industriel, qui permet de rémunérer les actionnaires.
Au demeurant, on a constaté un effet de dépendance (Pierson, 2000) autour de la politique énergétique nationale qui, historiquement, a accordé un poids prépondérant au nucléaire civil. En effet, les choix passés en matière d'énergie "limitent ainsi les possibilités de sortir d'une société carbonée » (La Branche, 2011) et conditionnent les orientations prises par la transition énergétique. Dans cette configuration, le processus «transitionnel » n'engendre pas de rupture nette avec le cadre actuel en inscrivant l'économie de marché dans une dynamique de décarbonisation.

\section{Remerciements}

Cette recherche a été menée grâce au soutien du Fonds européen de développement régional (FEDER), du programme Interreg IV Rhin supérieur. Cette recherche est le fruit d'une collaboration avec l'Institut des Sciences de l'environnement de l'université de Koblenz-Landau, l'Institut de Science régionale de l'université de Karlsruhe, ainsi que le Laboratoire Image, ville, environnement (LIVE), UMR 7362 CNRS-université de Strasbourg. Enfin le volet sociologique de l'étude a été porté par une équipe du Laboratoire Société, acteurs et gouvernements en Europe (SAGE) UMR 7363 CNRSuniversité de Strasbourg animée par Philippe Hamman, Guillaume Christen, Isabelle Hajek et Maurice Wintz. Voir le site Internet dédié : [http:// www.plan-ee.eu/].

\section{Bibliographie}

Adler F., Schachtschneider U., 2010. Green New Deal, Suffizienz oder Ökosozialismus? Konzepte für gesellschaftliche Wege aus der Ökokrise, München, Oekom, 318 p.

Aкrich M., 1993. Les formes de la médiation technique, Réseaux, $\mathrm{n}^{\circ} 60$, p. 87-98.

Aкrich M., 2006. La construction d'un système sociotechnique. Esquisse pour une anthropologie des techniques, in Akrich M., Callon M., Latour B. (dir.), Sociologie de la traduction, textes fondateurs, Paris, Presses des Mines, p. 109-134.

Akrich M., Méadel C., 1999. Histoire des usages modernes, in Beltran A., Akrich M., Méadel C., Duclos D. (dir.), Énergie, l'heure des choix, Paris, Éditions du Cercle d'Art, p. 25-91. 
Alter N., 1996. Sociologie de l'entreprise et de l'innovation, Paris, PUF, 241 p.

Brives H., Mormont M., 2008. La médiation de l'action collective " environnementale », in Françors M. (dir.), Écologisation. Objets et concepts intermédiaires, Bruxelles, PIE-Peter Lang, p. 129-137.

Christen G., Hajek I., Hamman P., Jehling M., Wintz M., 2013. «Quels enjeux à l'introduction des énergies renouvelables? Une analyse comparative Alsace/RhénaniePalatinat », Revue d'Allemagne et des Pays de langue allemande, 45 (1), p. 83-108.

Christen G., Hamman P., Jehling M., Wintz M. (dir.), 2014. Systèmes énergétiques renouvelables en France et en Allemagne. Synergies et divergences, Paris, Orizons, 331 p.

Coulbaut-Lazzarini A., Némoz S., 2013. L'éco-innovation au prisme du développement durable, Paris, L'Harmattan, 130 p.

Coutard O., 2001. Imaginaire et développement des réseaux techniques, Réseaux, 109, p. 76-94.

Coutard O., LÉvy J., 2010. Écologies urbaines, Paris, AnthroposÉconomica, 317 p.

Coutard O., Rutherford J., 2009. « Les réseaux transformés par leurs marges : développement et ambivalence des techniques "décentralisées" », Flux, 76-77, p. 6-13.

Debourdeau A., 2011. De la « solution » au "problème ». Les problématisations de l'obligation d'achat de l'énergie solaire photovoltaïque en France et en Allemagne, Politix, 95, p. 103-127.

Debourdeau A., 2011. Domestiquer le solaire. Transformations des modes de consommation et de production de l'électricité : l'exemple de la mise en marché du photovoltaïque, in Sandrine B., Emmanuel K., (dir.), Consommer et protéger l'environnement. Opposition ou convergence?, Paris, L'Harmattan, p. 47-66.

Évrard A., 2014. Les énergies renouvelables et l'électricité. À propos d'un conflit entre un secteur et une alternative de politique publique, Écologie et politique, 49, p. 67-80.

Gibson J., 1979. The Ecological Approach to Visual Perception, Boston, Houghton Mifflin, 332 p.

Gras A., 2003. « Les réseaux, les machines et la mégamachine : sur l'origine des systèmes techniques contemporains », in Musso P., Réseaux et société, Paris, PUF, p. 141-152.

Gras A., 2007. Le choix du feu. Aux origines de la crise climatique, Paris, Fayard, 281p.

Grolleau G., Mzoughi N., Thiébaut L., 2008. « Les “alliances vertes" entre les entreprises et les associations de protection de l'environnement : une réelle réconciliation ou une "instrumentalisation” réciproque? ", Revue d'économie régionale et urbaine, 4, p. 617-633.

Hubert B., Mormont M., 2008. De l'environnement au développement durable. Le rôle des médiateurs, in François M. (dir.), Écologisation. Objets et concepts intermédiaires, Bruxelles, PIE-Peter Lang, p. 51-68.
Huber J., 1982. Die verlorene Unschuld der Ökologie. Neue Technologien und superindustrielle Entwicklung, Frankfurt am Main, Fischer Verlag, 232 p.

La Branche S., 2011. « La méta-gouvernance climatique estelle soutenable dans le développement durable? ", in LA Branche S. (dir.), Le changement climatique : du métarisque à la méta-gouvernance, Paris, Tec \& Doc, p. 181-205.

Lafaye C., Thévenot L., 1993. «Une justification écologique? Conflits dans l'aménagement de la nature », Revue française de sociologie, 34 (4), p. 495-524.

Lascoumes P., Le Galès P., 2005. Gouverner par les instruments, Paris, Presses de Sciences Po, 370 p.

Nonjon M., 2005. «Professionnels de la participation : savoir gérer son image militante », Politix, 70, p. 89-112.

Pierson P., 2000. « Path Dependence, Increasing Returns, and the Study of Politics ", American Political Science Review, 94 (2), p. 251-267.

Poize N., Rüdinger A., 2014. Projets citoyens pour la production d'énergie renouvelable : une comparaison FranceAllemagne, working papers IDDRI, 1. [http://www.iddri. org/Publications/Collections/Idees-pourledebat/WP0114_ NP\%20AR_projets\%20citoyens.pdf].

Raineau L., 2011 . Adaptation aux changements climatiques. Vers une transition énergétique, Natures Sciences Sociétés, 19 (2), p. 133-143.

Rudolf F., 2013. De la modernisation écologique à la résilience : un réformisme de plus?, VertigO - la revue électronique en sciences de l'environnement, 13 (3) DOI : 10.4000/ vertigo. 14558.

Rumpala Y., 2013. Formes alternatives de production énergétique et reconfigurations politiques. La sociologie des énergies alternatives comme étude des potentialités de réorganisation du collectif, Flux, 92, p. 47-61.

Rutherford J., 2013. Avant-propos. Les flux d'énergie, Flux, 93-94, p. 4-6.

SCARwEll H., 2012. Éditorial : Rio+20, dépasser l'horizon de la conférence, Développement durable et territoires, 3 (2), [http://developpementdurable.revues.org/9341].

Theys J., 2014. "Le développement durable face à sa crise : un concept menacé, sous-exploité ou dépassé? ", Développement durable et territoires, 5 (1). [http://developpementdurable.revues.org/10196].

Werner-Brand K., 2014. "Sustainability transition". La durabilité, défi social et politique, in Charles L., Lange H., Kalaora B., Rudolf F. (dir.), Environnement et sciences sociales en France et en Allemagne, Paris, L'Harmattan, p. 117-146.

Zaccai E., 2010. "L'échec de Copenhague en perspective », Esprit, 362, p. 6-13.

ZÉLEM M.-C., 2012. Les énergies renouvelables en transition : de leur acceptabilité sociale à leur faisabilité sociotechnique, Revue de l'énergie, 6 (10), p. 418-424. 